\title{
The organic universe
}

\author{
Our inventory of the molecular universe is continually progressing. Our understanding of the astrochemistry behind \\ it will flourish if we are mindful of funding experimental and theoretical efforts as well as observational.
}

$\mathrm{R}$ oughly $5 \%$ of the Universe is composed of atoms, and of that $5 \%$, hydrogen and helium atoms make up the bulk. Of the remaining $0.1 \%$, half comprises oxygen atoms and a quarter is carbon. All the other elements are squeezed into the remaining $0.025 \%$, including chlorine at $0.0005 \%$. It is surprising then that if we look at the list of the $\sim 200$ molecules discovered in space, in the circumstellar and interstellar media, that organic molecules (roughly meaning those containing carbon) dominate. These range in size from the first species ever discovered in space, methylidyne $(\mathrm{CH}$, in 1937$)$ to the largest molecule thus far detected, $\mathrm{C}_{70}$, a buckyball (in 2010), and includes alcohols, primitive sugars, amino acids and more. The versatility of carbon in its chemical bonding drives the complexity of the chemistry in the Universe.

In this October issue of Nature Astronomy, we announce a development in our knowledge of this chemical complexity. Consistently we are discovering new chemical species in space at the rate of four per year. However, here we reveal not only the detection of a new molecule, but also that this molecule is the first molecule in a new family of extraterrestrial molecules. Edith Fayolle and colleagues from the ALMA-PILS (Protostellar Interferometric Line Survey) team, along with Kathrin Altwegg and the ROSINA team (article no. 0237), have detected the molecule chloromethane $\left(\mathrm{CH}_{3} \mathrm{Cl}\right)$, an organohalogen. There are also hints in the ALMA data of a further organohalogen, fluoromethane. It is the circumstances in which chloromethane has been found that add interest to the situation: two separate detections, one around a forming star, and another in a comet in a mature planetary system, our own. Connecting the dots (as Marcelino Agúndez does in his accompanying News \& Views (article no. 0265)), we must ask whether chloromethane and other organic molecules can survive the star- and planet-formation process, preserved carefully on icy bodies such as comets and Kuiper belt objects. It seems possible. The logical follow-up question to this is whether organic molecules can then be delivered intact by comets to terrestrial planets through impacts, and here there is still work to be done. However, the consequences are important because they may have implications for the development of life on early Earth.
When we think about organic molecules in space, and particularly prebiotic molecules such as amino acids and simple sugars, our thoughts inevitably turn towards the possibility of life in the wider Universe. The only known examples of life are found on our planet, but of course one of the big questions of our technological generation is whether we might detect signs of life elsewhere, and intelligent life in particular. This question was recently posed to the team behind the Voyager mission, in a cinematic documentary entitled The Farthest (read our review of this film in this month's Books \& Arts section; article no. 0274). All of them were positive that life must exist elsewhere in the Universe: statistically it is extremely likely. There are many crucial factors that can play a role, though, among which coincidental timescales is perhaps the most staggering as a race, will the fleetingly short period in cosmic history that belongs to us overlap with that of another race?

However, we should be careful not to equate 'organic molecules' with 'life', since as Sun Kwok points out in his Comment (article no. 0272), the vast majority of organic molecules in the Universe arise from an abiotic formation pathway and will not lead to genesis. Nevertheless, they are still an important part of the chemical cosmos. We already know that the 'unidentified infrared emission' bands seen almost ubiquitously in the Universe are likely carried by hydrocarbons, although whether these are polycyclic aromatic hydrocarbons or mixed aromatic/aliphatic organic nanoparticles is still not settled (or indeed, whether these two terms, in common usage, mean the same thing). These carriers alone may consume up to $20 \%$ of the cosmic carbon. The 'diffuse interstellar bands' (DIBs), also ubiquitous, are probably also carried by carbonaceous molecules: the only identified carrier to date is $\mathrm{C}_{60}{ }^{+}$(E. K. Campbell, M. Holz, D. Gerlich \& J. P. Maier, Nature 523, $322-323 ; 2015)$, which is responsible for (at least) two of the hundreds of DIBs.

The ALMA telescope is blowing open the doors to the molecular universe: we've seen examples of its fruit in the pages of Nature Astronomy already, in objects near

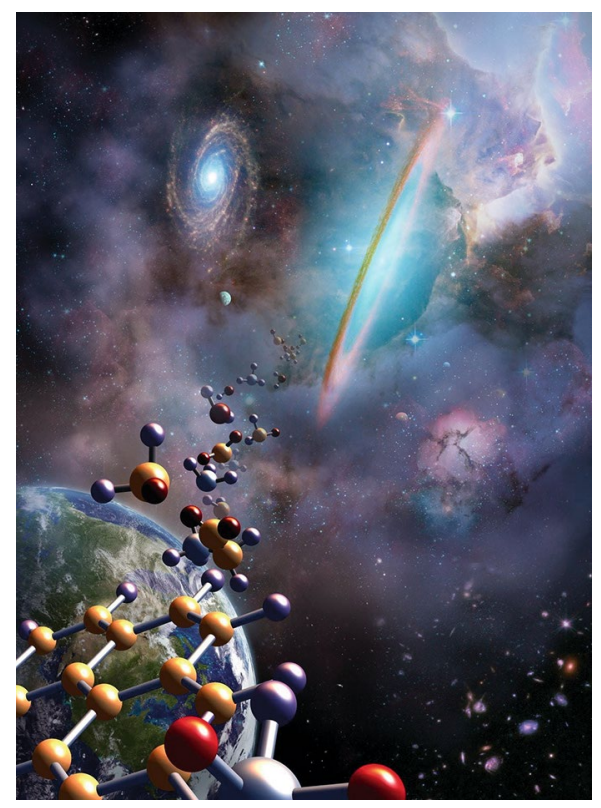

Credit: NASA / Jenny Mottar

(protostars, evolved stars, molecular clouds near black holes) and far (intermediate- and high-redshift galaxies). It's a fantastic facility that promises to keep on delivering, especially with the continuing developments in instrumentation, such as the one we covered in our June Mission Control (article no. 0156).

But we should bear in mind that astronomical detections are only part of the picture. They rely on accurate and detailed laboratory spectra, or intensive computations. A detection is only the tip of the iceberg in understanding the underlying astrochemistry: How does a chemical species form in space? How is it destroyed? How long does it live? What can it tell us about the conditions in which it exists? Challenging laboratory experiments and comprehensive chemical models and databases are needed to fully reveal what lies under the surface. So while ALMA is deservedly grabbing the science headlines, we should be mindful in funding the labs and computers and people that will more deeply unveil the richness of our complex chemical cosmos, for they are often more hidden from view.

Published online: 3 October 2017 DOI: 10.1038/s41550-017-0283-1 